\title{
Tumor Lysis Syndrome with Recurrence in a Patient with Multiple Myeloma Following Bortezomib Therapy - A Case Report
}

Kemarut C. Laothamatas, MD, Indira Laothamatas, BA, and Bilal Asif, MD

\section{INTRODUCTION}

Tumor lysis syndrome (TLS) is a life-threatening complication most commonly observed in highly proliferative hematologic malignancies. The rapid release of intracellular contents causes hyperkalemia, hyperuricemia, hyperphosphatemia, and secondary hypocalcemia. The constellation of metabolic abnormalities can manifest clinically as renal insufficiency, cardiac arrhythmias, seizures, and death due to multi-organ failure. Traditionally, TLS is rarely observed in relatively indolent malignancies such as multiple myeloma. However, its frequency may be increasing with the advent of more effective and targeted therapies such as bortezomib. We herein report a 62-year-old man with IgA kappa light chain multiple myeloma who developed two episodes of TLS ten days apart following a single cycle of bortezomib therapy.

\section{CASE PRESENTATION}

A 62-year-old man presented to a local community hospital with a month of progressive back pain. He was found to have T11-L4 pathological fractures and was subsequently found to have an elevated kappa-to-lamba ratio at 1606 with IgA predominance on the SPEP. Flow cytometry of the peripheral blood was negative for plasmablasts. He soon underwent a bone marrow biopsy with aspirate revealing greater than $90 \%$ plasma cell involvement of the bone marrow. Based on the workup, he was diagnosed with IgA kappa light chain multiple myeloma and was initiated on bortezomib therapy. He was deemed stable for discharge on the same day that the therapy was initiated given no acute adverse reactions were observed. Within 24 hours, he became acutely altered and was readmitted. He was intubated for airway protection and given intravenous hydration and broad-spectrum antibiotics prior to being transferred to our medical intensive care unit. On arrival, he was anuric and in acute renal failure. His presentation and laboratory findings met criteria for both clinical and laboratory TLS (Table 1, Figure 1). He was treated with rasburicase and started on hemodialysis. His TLS labs gradually normalized. Broad-spectrum antibiotics were continued through Day 6 of his hospitalization ( 8 days after bortezomib therapy).
On Day 9 of his hospitalization (11 days after bortezomib therapy), wide complex tachycardia was noted on telemetry. He was febrile with a maximum temperature of $104^{\circ} \mathrm{F}$. Repeat laboratory work confirmed a recurrence of TLS including marked hyperkalemia of $8.6 \mathrm{mmol} / \mathrm{L}$ despite the patient having received hemodialysis earlier that day (Table 1, Figure 1). His labs were also remarkable for new leukocytosis and severe metabolic acidosis. He was promptly treated with rasburicase and broadspectrum antibiotics were restarted. He received medical treatment for hyperkalemia and was initiated of continuous venovenous hemodialysis. Yet his telemetry continued to show wide complex tachycardia, which evolved into ventricular fibrillation and cardiac arrest. Advanced cardiovascular life support was performed with return of spontaneous circulation, but he remained in tenuous condition with marked hypotension and diffuse bleeding. His family decided to initiate comfort measures, and he expired shortly thereafter.

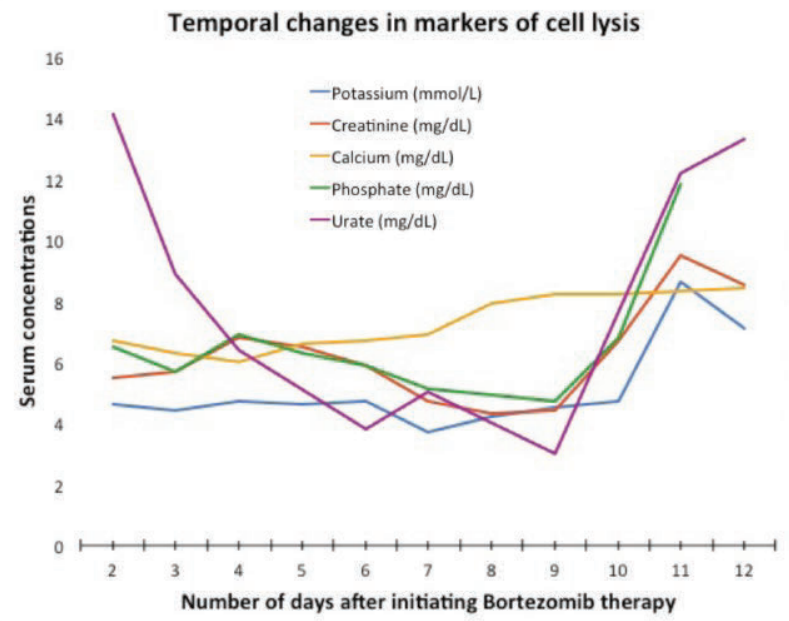

Figure 1. Temporal changes in potassium, creatinine, calcium, phosphate, and urate concentrations in our patient with IgA kappa light chain multiple myeloma after receiving bortezomib therapy. 
Table 1: Laboratory values in our patient with IgA kappa light chain multiple myeloma after receiving bortezomib therapy.

\begin{tabular}{|c|c|c|c|c|c|c|c|}
\hline $\begin{array}{l}\text { Number of days } \\
\text { after initiating } \\
\text { bortezomib therapy }\end{array}$ & $\begin{array}{l}\text { Potassium } \\
\text { (mmol/L) }\end{array}$ & $\begin{array}{l}\text { Creatinine } \\
\text { (mg/dL) }\end{array}$ & $\begin{array}{l}\text { Calcium } \\
\text { (mg/dL) }\end{array}$ & $\begin{array}{l}\text { Phosphate } \\
\text { (mg/dL) }\end{array}$ & $\begin{array}{l}\text { Urate (mg/ } \\
\text { dL) }\end{array}$ & LDH (IU/L) & INR \\
\hline 2 & 4.6 & 5.5 & 6.7 & 6.5 & 14.1 & 2011 & 2.52 \\
\hline 3 & 4.4 & 5.7 & 6.3 & 5.7 & 8.9 & $\mathrm{~N} / \mathrm{A}$ & 2.1 \\
\hline 4 & 4.7 & 6.8 & 6 & 6.9 & 6.4 & 1229 & 1.85 \\
\hline 5 & 4.6 & 6.5 & 6.6 & 6.3 & $\mathrm{~N} / \mathrm{A}$ & N/A & 1.65 \\
\hline 6 & 4.7 & 5.9 & 6.7 & 5.9 & 3.8 & 240 & 1.59 \\
\hline 7 & 3.7 & 4.7 & 6.9 & 5.1 & 5 & 197 & 1.61 \\
\hline 8 & 4.2 & 4.3 & 7.9 & $\mathrm{~N} / \mathrm{A}$ & $\mathrm{N} / \mathrm{A}$ & N/A & 1.37 \\
\hline 9 & 4.5 & 4.4 & 8.2 & 4.7 & 3 & $\mathrm{~N} / \mathrm{A}$ & 1.36 \\
\hline 10 & 4.7 & 6.7 & 8.2 & 6.8 & $\mathrm{~N} / \mathrm{A}$ & $\mathrm{N} / \mathrm{A}$ & 1.36 \\
\hline 11 & 8.6 & 9.5 & $\mathrm{~N} / \mathrm{A}$ & 11.8 & 12.2 & 353 & 1.6 \\
\hline 12 & 7.1 & 8.5 & 8.4 & $\mathrm{~N} / \mathrm{A}$ & 13.3 & 848 & 1.99 \\
\hline
\end{tabular}

\section{FOLLOW-UP}

His autopsy revealed pneumonia at the time of death. Blood cultures drawn shortly before the patient expired grew Pseudomonas aeruginosa and coagulase-negative Staphylococcus.

\section{DISCUSSION}

Tumor lysis syndrome (TLS) has been reported in patients with a variety of diagnoses but its incidence is greatest in highly proliferative hematologic malignancies such as acute lymphocytic lymphoma or Burkitt's lymphoma (5.2-23\% and $14.9 \%$, respectively). ${ }^{1}$ In contrast, this condition is usually rare in patients with multiple myeloma. The following disease characteristics have been shown to be associated with the development of TLS in these patients: immature plasma cell morphology, heavy plasma cell burden within the bone marrow (plasma cells $>70 \%$ of all nucleated cells), high proliferative activity of plasma cells and cytogenetic abnormality within chromosome 11 or $13 .{ }^{2}$ Our patient had a heavy plasma cell burden within the bone marrow based on his bone marrow biopsy on his last admission.

Bortezomib is a proteasome inhibitor approved in the US for treating multiple myeloma and mantle cell lymphoma. It induces cell death by preventing the degradation of pro-apoptotic proteins. Officially, bortezomib is thought to be associated with TLS, but whether or not the therapy induces TLS is debatable. ${ }^{3}$ Based on a retrospective study conducted before the approval of bortezomib, $1 \%$ of over 800 multiple myeloma patients treated with other modalities including cyclophosphamide, dexamethasone, etoposide, and stem cell transplants developed TLS. This number is strikingly similar to the $1.4 \%$ of 496 multiple myeloma patients who developed TLS following bortezomib treatment. ${ }^{4}$ Both studies found an association between the development of TLS in multiple myeloma patients and the aforementioned high-risk disease characteristics. Therefore, it may be that the incidence of TLS is more strongly associated with this patient population than with the use of bortezomib itself.

As far as we know, all cases of TLS following bortezomib therapy have occurred within the first cycle of treatment. ${ }^{5}$ Furthermore, per our literature search, recurrence of TLS within a single cycle of treatment has never been described. Our patient developed the first occurrence of TLS within 24 hours of bortezomib administration. Following treatment with rasburicase and hemodialysis, his TLS labs completely normalized. However, several days later, he developed a presumed second episode of TLS with no new known risk factors. The recurrence of TLS coincided with a new leukocytosis and bacteremia. The relationships between previous episodes of TLS, concomitant bacteremia, and the recurrent development of TLS are unknown.

It may be worthwhile to consider TLS in the differential diagnosis of hyperkalemia and wide complex tachycardia in multiple myeloma patients. In our case, the diagnosis of TLS did not promptly come to mind since recurrence of TLS is unusual. We believe that it is important to distinguish TLS-associated hyperkalemia from other etiologies of hyperkalemia such as acute renal failure or severe acidosis as the two would require different treatment regimens. 


\section{CONCLUSION}

The incidence of TLS is expected to rise with the advent of more effective and targeted therapies. Currently, there is no official guideline for TLS prophylaxis in multiple myeloma patients receiving bortezomib. However, clinicians should be aware of the possibility of TLS developing within the first cycle of treatment - especially in patients with high-risk disease characteristics - and consider administering appropriate prophylaxis, including IV hydration, allopurinol or even treating with rasburicase, on a case-by-case basis. Furthermore, based on our case report, renal insufficiency, concomitant infection, and a previous episode of TLS may serve as additional risk factors and thus warrant further investigation.

\section{REFERENCES}

1. Wilson FP and Berns JS. Tumor lysis syndrome: new challenges and advances. Advances in Chronic Kidney Disease. 2014; 21(1):18-26

2. Fassas ABT, Desikan KR, Siegel D, Golper TA, Munshi NC, Barlogie B, Tricot G. Tumour lysis syndrome complicating high-dose treatment in patients with multiple myeloma. British Journal of Haematology. 1999;105: 938-941.

3. Millennium Pharmaceuticals. Velcade (Bortezomib) for injection [summary of product characteristics]. Cambridge MA: Millennium Pharmaceuticals; 2008

4. Sezer O, Vesole DH, Singhal S, Richardson P, Stadtmauer E, Jakob C, Boral AL, Esseltine DL, Mehta J. Bortezomib-induced tumor lysis syndrome in multiple myeloma. Clinical Lymphoma, Myeloma \& Leukemia. 2006;7(3):233-235.

5. Furtado M, Rule S. Bortezomib-associated tumor lysis syndrome in multiple myeloma. Leukemia \& Lymphoma. 2008;49(12):2380-2382.

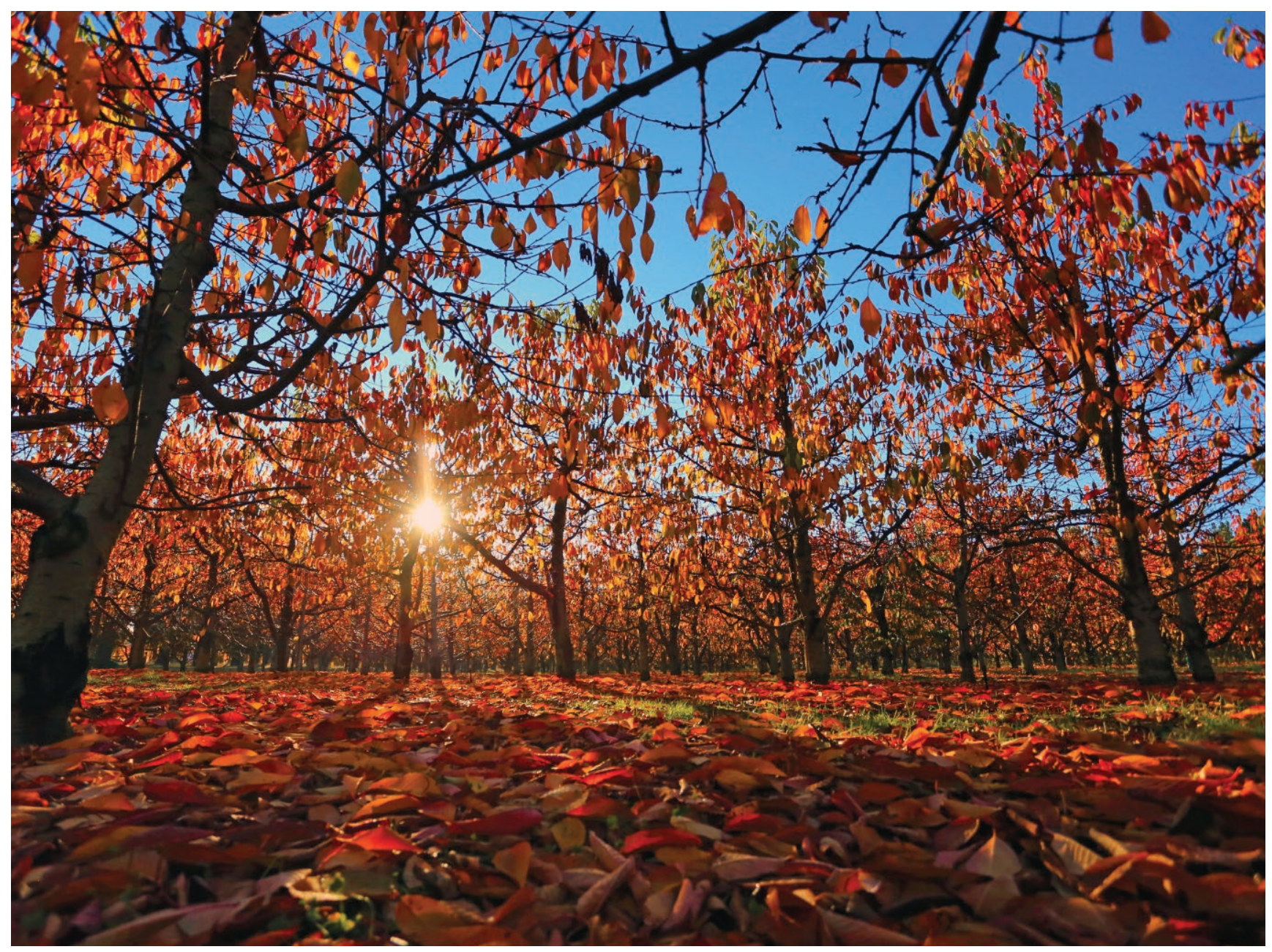

